\title{
Urotensin II Induces Cardiac Fibrosis through the TGF- $\beta /$ Smad Signaling Pathway during the Development of Cardiac Hypertrophy
}

\author{
Yanyan Liang, ${ }^{1 *} \mathrm{MD}$, Yifeng $\mathrm{Xu},{ }^{1 *} \mathrm{MD}$, Lin Ding, ${ }^{1} \mathrm{MD}$, Xiaoqing Chen, ${ }^{1} \mathrm{MD}$ and Hongli $\mathrm{Li},{ }^{1} \mathrm{MD}$
}

\begin{abstract}
Summary
Myocardial fibrosis is an important pathological phenomenon of cardiac remodeling that is induced by hypertension, myocardial ischemia, valvular heart disease, hypertrophic cardiomyopathy, and other heart diseases and can progress to heart failure. Urotensin II (UII) is regarded as a cardiovascular autacoid/hormone that is not only the most potent vasoconstrictor in mammals but also involved in cardiac remodeling. However, the molecular mechanisms responsible for UII-induced cardiac fibrosis have not yet been fully elucidated. Therefore, we aimed to investigate the effect of UII on myocardial fibrosis in cardiac hypertrophy and the mechanism of UII-induced cardiac fibrosis. Cardiac tissue from mice subjected to Transverse aortic constriction (TAC) was collected. Cardiac hypertrophy, myocardial fibrosis, and the expression of UII protein were assessed using echocardiography and pathological and molecular biological analyses. The effect of UII on fibrosis was evaluated in UII-treated mice and isolated rat primary cardiac fibroblasts, and the results indicated that UII induced significant myocardial fibrosis and increases in the proliferation and fibrotic responses both in mice and cultured fibroblasts. Mechanistically, UII treatment induced activation of the TGF- $\beta /$ Smad signaling pathway, which was suppressed by the UII receptor antagonist. In conclusion, UII plays critical roles in cardiac fibrosis by modulating the TGF- $\beta /$ Smads signaling pathway, which may be a promising therapeutic target in hypertrophic cardiomyopathy and related problems, such as cardiac remodeling and heart failure.
\end{abstract}

Key words: UII, Myocardial fibrosis, Fibroblasts

(Int Heart J 2021; 62: 1135-1144)

$\mathrm{H}$ ypertrophic cardiomyopathy (HCM) is a common type of hereditary cardiomyopathy, typically of autosomal recessive inheritance, that is characterized by myocardial asymmetric hypertrophy, limited left ventricular filling, a smaller ventricular cavity, and a decrease in diastolic compliance. $\left.{ }^{1,2}\right) \mathrm{HCM}$ is the main cause of sudden cardiac death (SCD), a sudden unexpected death caused by loss of heart function. The annual mortality rate of HCM is roughly $1 \%$, and the worldwide incidence is approximately $0.2 \% .^{1,3)}$ Myocardial fibrosis is a typical pathological change that is often observed in patients with HCM. Simultaneously, myocardial fibrosis includes a variety of clinical symptoms and is closely related to the prognosis. HCM myocardial fibrosis, especially scar formation, is considered an important aspect of heart failure. ${ }^{4)}$ Therefore, studies that focus on the mechanism of action, causal factors, and treatment of HCM myocardial fibrosis have become a hotspot in HCM research.

Urotensin II (UII), a cyclic neuropeptide originally isolated from the fish spinal cord ${ }^{5,6)}$ has been recently detected and found to be functionally implicated in mammalian central nervous and cardiovascular systems. ${ }^{6-8)}$ In the cardiovascular system, many reports indicated that UII might be implicated in cardiovascular regulation, with promising therapeutic applications based on UT receptor antagonism. ${ }^{9)}$ UII has strong effects on vascular activity and can induce contraction of rat thoracic aorta ${ }^{6}$ and dilate human pulmonary artery, and reduce abdominal vascular resistance ${ }^{10)}$ Moreover, UII administration to human subjects induced ST segment changes in the electrocardiogram observed in myocardial ischemia. ${ }^{6}$ UII is widely expressed in the cardiovascular system, with evidence for expression of a UII protein or gene in vascular smooth muscle cells, endothelial cells, endocardial endothelial cells, inflammatory cells, cardiomyocytes, and fibroblasts. ${ }^{6-9,11-15)}$ These findings provide strong evidence for transcriptional and translational regulation of the UII system locally within the cardiovascular system. In a physical state, low levels of UII could be detected in plasma, supporting the hypothesis that it acts as an autocrine and/or paracrine agent. In response to cardiovascular risk factors and pathological changes such as hypertension, obesity, diabetes, aging, atherosclerosis, cardiac hypertrophy, and heart failure, the expression of UII could be increased not only in the cardiovascular system but also in plasma, sug-

From the ${ }^{1}$ Department of Cardiology, Shanghai General Hospital, School of Medicine, Shanghai Jiao Tong University, Shanghai, China.

* These authors contributed equally to this work.

Address for correspondence: Hongli Li, MD, Department of Cardiology, Shanghai General Hospital, School of Medicine, Shanghai Jiao Tong University, 100 Haining Rad, Hongkou District, Shanghai 200080, China. E-mail: drhonglili@126.com

Received for publication January 13, 2021. Revised and accepted May 6, 2021.

doi: 10.1536/ihj.21-032

All rights reserved by the International Heart Journal Association. 
Table I. Echocardiography and Pathology of PBS- and UIITreated Groups

\begin{tabular}{lcc}
\hline & PBS & UII \\
\hline 4 weeks & & \\
Echocardiography, $n$ & 9 & 20 \\
LVID; d, mm & $3.73 \pm 0.24$ & $4.0525 \pm 0.40$ \\
LVPW; d, mm & $0.67 \pm 0.08$ & $0.86 \pm 0.18^{*}$ \\
FS\% & $43.81 \pm 1.18$ & $36.78 \pm 6.27$ \\
EF\% & $71.11 \pm 11.38$ & $70.45 \pm 9.11$ \\
SBP $(\mathrm{mmHg})$ & $112.3 \pm 10.8$ & $117.7 \pm 14.1^{*}$ \\
Pathology, $n$ & 9 & 20 \\
BW, g & $24.52 \pm 1.97$ & $22.58 \pm 1.62$ \\
HW, mg & $123.69 \pm 15.67$ & $136.69 \pm 25.09$ \\
TIBIA, mm & $12.90 \pm 0.58$ & $11.78 \pm 1.03$ \\
HW/TIBIA, mg/mm & $9.6 \pm 0.34$ & $11.82 \pm 2.61^{*}$ \\
HW/BW, mg/g & $5.05 \pm 0.25$ & $5.45 \pm 0.51^{*}$ \\
\hline
\end{tabular}

BW indicates body weight; $\mathrm{EF}$, ejection fraction; FS, fractional shortening; SBP, systolic blood pressure; HW/TIBIA, heart weight/tibia length ratio; LVID; d, left ventricular internal diastolic dimension; and LVPW; d, left ventricular diastolic posterior wall. $* P<0.05$ versus PBS.

gesting the pathophysiological roles of UII in cardiovascular diseases..$^{6-9,11-18)}$ The expression of UII has been demonstrated to be increased in cardiac tissue from patients with congestive heart failure, which positively correlates with disease severity and inversely with cardiac function. ${ }^{16)} \mathrm{Re}$ cently, serum UII level has been reported to be markedly elevated in patients with hypertrophic cardiomyopathy and acute myocardial infarction, ${ }^{17,18)}$ suggesting that UII might be a novel biomarker parameter that has clinical use in patients with these diseases.

Actions of UII in mammals are proposed to be mediated through interactions with certain somatostatin receptor subtypes.9) An orphan human G protein-coupled receptor homologous to rat GPR14 has been found to be expressed predominantly in cardiovascular tissues and to function as a receptor for UII. ${ }^{69)}$ It has been certified that UII-induced cardiac hypertrophy involves multiple signaling pathways, including mitogen-activated protein kinase signaling pathways, extracellular signal-regulated kinase, epidermal growth factor receptor, and Akt and $\mathrm{Ca}^{2+}$ relative signaling pathways. ${ }^{9)}$ However, the precise signaling mechanisms responsible for UII-induced cardiac remodeling remain to be elucidated. Since cardiac fibrosis plays an important role in ventricular remodeling, we investigated the effect of UII on cardiac fibrosis. Our data indicated that UII was involved in the myocardial fibrosis during the development of cardiac hypertrophy through regulation of the TGF- $\beta /$ Smad signaling pathway. These findings provide a novel powerful role of UII in cardiac muscle tissue and demonstrate a new potential target for the treatment of cardiac remodeling.

\section{Methods}

Animals and in vivo studies: Male C57BL/6 mice (22-25 g) were obtained from Shanghai Laboratory Animal Center, Chinese Academy of Sciences (Shanghai, China), and were randomly divided into a control group and a UII (PEPTIDE INSTITUTE, INC., Osaka, Japan)-treated group. The UII was administrated to mice by subcutaneous injection with $1.5 \mu \mathrm{g} / \mathrm{kg}$ (once daily, lasting for 4 weeks), ${ }^{19-21)}$ since UII at this dose could induce significant cardiac hypertrophy without the elevation of blood pressure (Table I). Animals were fed a chow diet and housed in a 12-hour light/12-hour dark environment at $25^{\circ} \mathrm{C}$ in the animal facility of the Shanghai Jiaotong University (Shanghai, China). Studies were performed according to the Guidelines for the Care and Use of Laboratory Animals as published by the National Institutes of Health (NIH Publication 8523, revised in 1996) and approved by the Animal Care Committee of the Shanghai Jiaotong University (Shanghai, China).

Transverse aortic constriction: Transverse aortic constriction (TAC) was performed as previously described. ${ }^{19,20,22)}$ A longitudinal incision of 2 to $3 \mathrm{~mm}$ was made through the proximal sternum to allow access and visualization of the aortic arch. Subsequently, the transverse aorta was ligated between the innominate and left common carotid arteries with an overlaid 27-gage needle. The needle was immediately removed, leaving a discrete constriction that was uniform in size throughout the TAC group. Sham-operated animals underwent a similar TAC procedure; however, the ligature was not tied.

Echocardiography and blood pressure measurement: Echocardiography and systolic blood pressure (SBP) measurement were performed on animals that underwent TAC and UII treatment as previously described. ${ }^{19,20,22)}$ In brief, ultrasonography was performed using a VisualSonics 770 Echocardiography instrument (Toronto, Ontario, Canada). Left ventricular systolic function and thickness of the ventricular wall were measured using a $30 \mathrm{MHz}$ transducer. Hemodynamic parameters, including the left ventricular ejection fraction, ventricular septal thickness (IVSd), ventricular systolic ventricular septal thickness (IVS), left ventricular diastolic posterior wall thickness (LVPWd), and left ventricular systolic posterior wall thickness (LVPWs) were calculated. SBP was measured using a micronanometer catheter (Millar 1.4F, SPR 835, Millar Instruments, Inc.) that was inserted via the right common carotid artery into aorta. The transducer was connected to a Power Laboratory system (AD Instruments, Castle Hill, Australia), and SBP was recorded. At 4 weeks after TAC surgery or UII treatment, mice were subjected to echocardiography and SBP measurement. To limit the effects of different heart rates on the aortic pressure gradient and left ventricular function, the isoflurane flow rate that was used to anesthetize the mice during the ultrasound procedure was adjusted to maintain a heart rate of 400500 times per minute.

Histochemical staining: Mice were anesthetized with a high dose of chloral hydrate, and their hearts were excised. For global morphometry, hearts were perfused with phosphate-buffered saline (PBS), followed by $4 \%$ polyformaldehyde. For histological analysis, heart tissues were fixed in $10 \%$ formalin and embedded in paraffin in such a fashion that sections could be cut through the short axis of the heart, sectioned at a $5 \mu \mathrm{m}$ thickness and processed routinely to staining with hematoxylin and eosin (H-E), Masson trichrome, and Sirius red (SR). Cardiomyocyte hypertrophy and the level of collagen deposition were 
Table II. Primers for qPCR and Genotyping

\begin{tabular}{lll}
\hline Primers & Forward (5'-3') & Reverse (5'-3') \\
\hline M-ANP & GTGCGGTGTCCAACACAGAT & TCCAATCCTGTCAATCCTACCC \\
M-BNP & GAGGTCACTCCTATCCTCTGG & GCCATTTCCTCCGACTTTTCTC \\
M-UII & AAGCACGTCAGCTAACTGTTC & TGTTGGTGTGTCTACCAGACC \\
M-GAPDH & ACTCCACTCACGGCAAATTC & TCTCCATGGTGGTGAAGACA \\
R-UII & ACAAAAATCAAAGCACGTCAGC & TGAGAGCATTCACCCGTCTT \\
R-ANP & TTTCAAGAACCTGCTAGACCAC & GGTTGACTTCCCCAGTCCAG \\
R-BNP & CAAGATGGCACATAGTTCAAGC & ACAGCCCAAGCGACTGAC \\
R- $\beta-$-MHC & GAGCCTCCAGAGTTTGCTGAAGGA & TTGGCACGGACTGCGTCATC \\
R-Collagen I & CACTGCAAGAACAGCGTAGC & AAGTTCCGGTGTGACTCGTG \\
R-Collagen III & AGGGCAGGGAACAACTGATG & GGTCCCACATTGCACAAAGC \\
R-TGF- $\beta$ & GCCTGAGTGGCTGTCTTTTG & AAAGCCCTGTATTCCGTCTCC \\
R-Smad 3 & TGTCATCTACTGCCGCTTGTG & TGTTGAAGGCGAACTCACAG \\
R-GPADG & AACGACCCCTTCATTGACCTC & TCGCCGATCTCAATCTTCTT \\
\hline
\end{tabular}

ANP indicates atrial natriuretic peptide; BNP, brain natriuretic peptide; $\beta$-MHC, $\beta$-myosin heavy chain; UII, Urotensin II; and GAPDH, glyceraldehyde-3-phosphate dehydrogenase.

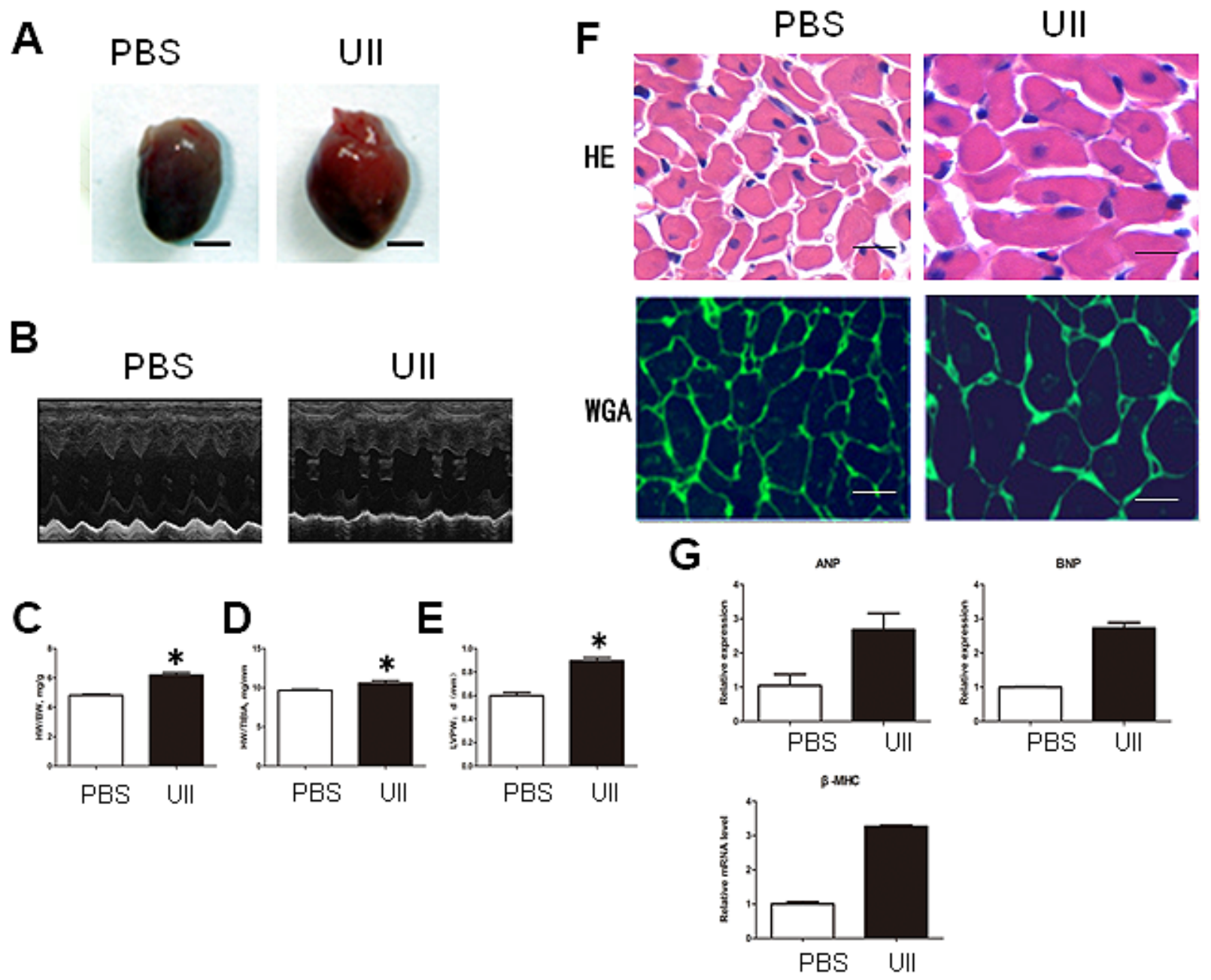

Figure 1. UII induces cardiac hypertrophy in mice. A: Representative images of the heart in mice 4 weeks after PBS and UII treatment (Scale Bar: $15 \mathrm{~mm}$ ). B: Representative images of the echocardiography in mice 4 weeks after PBS and UII treatment. C-E: The ratios of heart weight (HW) /mouse body weight (BW), heart weight (HW)/tibia length (TL), and left ventricular posterior wall (LVPW) comparison ( $n=10$ to 12 mice/ group) in PBS- and UII-treated mice at 4 weeks after treatment. F: Representative tissue sections of hearts with hematoxylin and eosin and WGA staining obtained from PBS- or UII-treated mice (magnification: $200 \times$ ). G: mRNA expression of hypertrophic markers in PBS- or UII-treated mice $(n=5$ mice/group). ANP indicates atrial natriuretic peptide; BNP, brain natriuretic peptide; and $\beta$-MHC, $\beta$-myosin heavy chain. $* P<0.05$ 

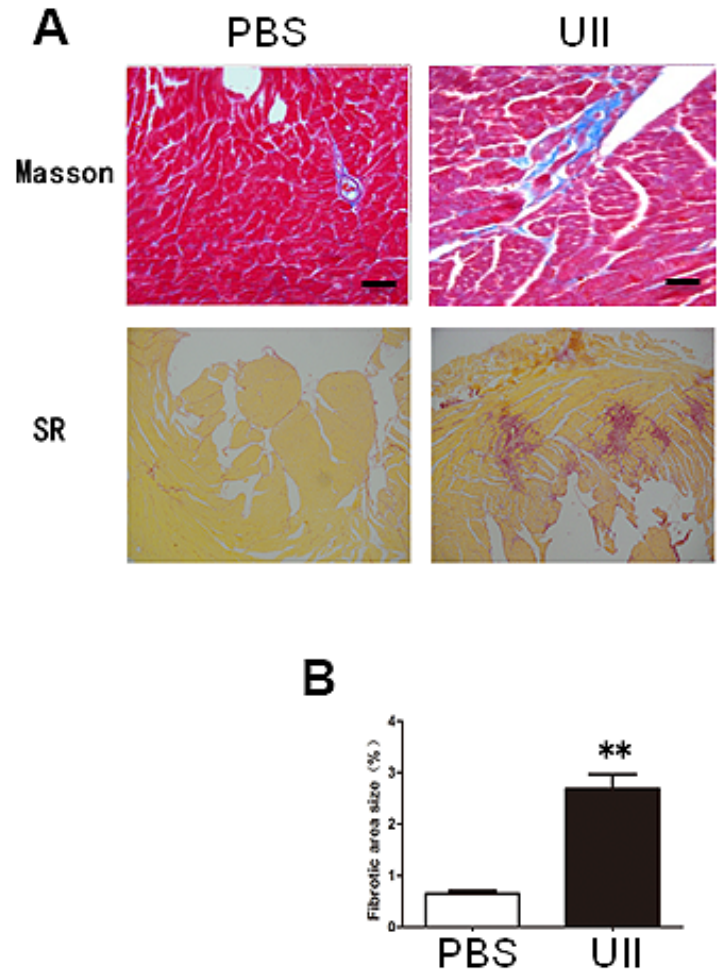
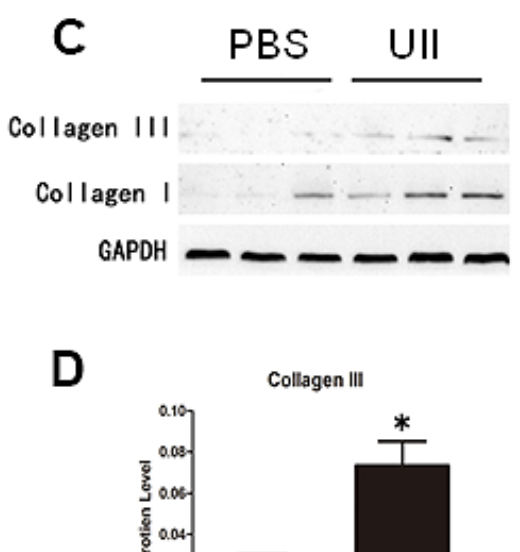

E
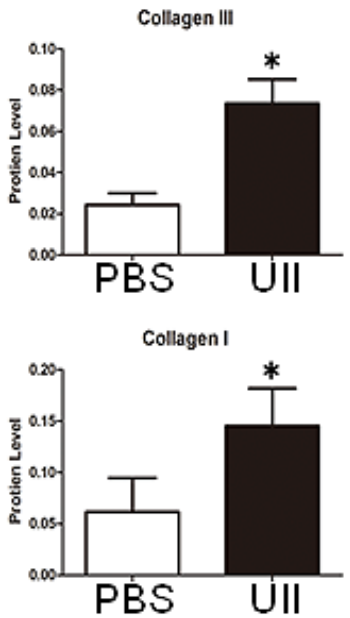

Figure 2. UII-induced cardiac fibrosis in mice. A: Representative images of Masson staining (magnification: $100 \times$ ) and Sirius red staining of mouse hearts 4 weeks after PBS- or UII-treatment (magnification: $40 \times$ ). B: Fibrosis area to total area radio from PBS- and UII-treated mice were quantified. C-E: Collagen I and collagen III expression levels in the left ventricle of PBS and UII-treated mice were quantified using Western blot analysis. PBS-treated mice: $n=9$, UII-treated mice: $n=9 . * P<0.05, * * P<0.01$

evaluated in five random high-power fields from each section and quantified in a blinded manner. Images were taken using an FSX100 microscope (Olympus Corporation, Tokyo, Japan). To quantify the degree of fibrosis, sections ( $n=9$ to 10$)$ were analyzed using Image-Pro Plus software (Media Cybernetics, Inc., Bethesda, MD, USA). The percentage of the fibrosis was calculated using the number of blue pixels per section divided by the total number of pixels present in the myocardium.

Immunohistochemical analysis : Paraffin-embedded mouse heart tissue was cut into $5 \mu \mathrm{m}$ sections, which were subjected to immunohistochemical staining. For fluorescein-conjugated wheat germ agglutinin (FITCWGA), sections were deparaffinized, rehydrated, rinsed with PBS, and incubated for 60 minutes in $50 \mathrm{mg} / \mathrm{ml}$ WGA-FITC (\#R-FWGA-001, Xian Ruixi Biological Technology Co., Ltd., Xian, China) in PBS. After three washing and coverslipping steps, sections were examined on an Olympus fluorescence microscope. For UII valuation in heart tissue, a UII antibody (mouse anti-UII antibody, ab 21630, Abcam) was used (1:200). Quantification of the UII-positive staining was determined on Image-Pro Plus 6.0 software using the staining area/total area.

Cell culture and treatments: Neonatal rat cardiomyocytes (NRCMs) and fibroblasts were isolated from the left and right ventricles of Sprague-Dawley rats on the second day after birth, essentially according to the methods of
Zou, et $a .^{23)}$ Trypsinization was performed with $0.05 \%$ trypsin-EDTA at $37^{\circ} \mathrm{C}$ for 10 minutes. To selectively enrich cardiac myocytes, dissociated cells were preplated onto $100 \mathrm{~mm}$ culture dishes for 30 minutes, which permitted preferential attachment of fibroblasts to the bottom of the dish. Nonadherent cells were plated at a field density of $5 \times 10^{5}$ cells $/ \mathrm{mL}$ on six-well culture dishes as an NRCM-rich culture. Cardiac fibroblasts were obtained from adherent cells on the preplating dish and split twice before use. NRCMs were cultured in Dulbecco's Modified Eagle's Medium (DMEM), supplemented with 10\% fetal bovine serum (FBS, Gibco, Carlsbad, CA, USA) at $37^{\circ} \mathrm{C}$ for 48 hours. Then, NRCMs were incubated with UII $(0.1$ $\mu \mathrm{M}$, PEPTIDE INSTITUTE, INC., Osaka, Japan) for 48 hours to induce cardiomyocyte hypertrophy responses. The induction of hypertrophy was evaluated by measuring cell hypertrophy-related gene expression $(\beta-\mathrm{MHC}$, atrial natriuretic peptide (ANP), and brain natriuretic peptide (BNP).

The obtained cardiac fibroblasts were grown to confluence and subsequently passaged 1:2 with trypsin. Cultures were incubated at $37^{\circ} \mathrm{C}$ in a humidified atmosphere of $5 \% \mathrm{CO}_{2} / 95 \%$ air. Studies were conducted on cardiac fibroblasts (passages 2-4) that were grown to subconfluence in serum-containing media for 48 hours and growth arrested for 24 hours in a serum-free medium before treatment. Cardiac fibroblasts were treated with UII $(0.1 \mu \mathrm{M})$ 
A
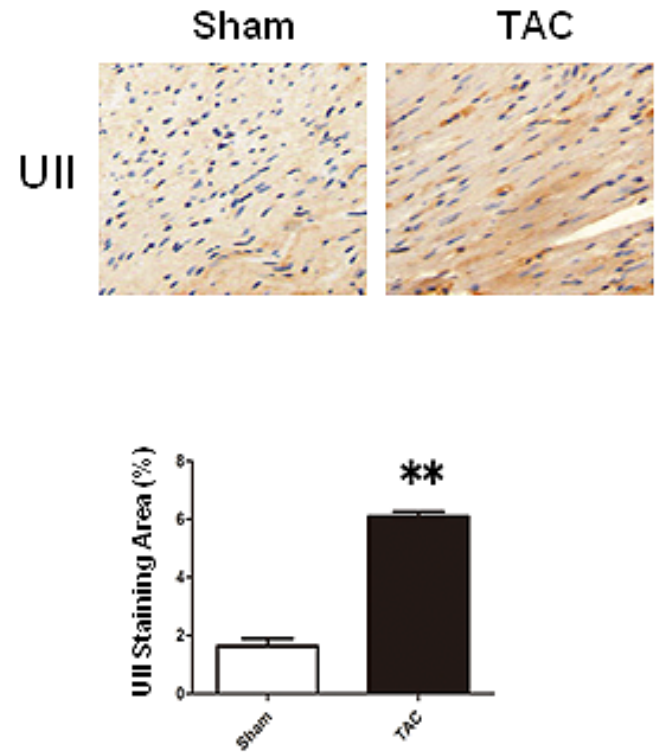

E

UII
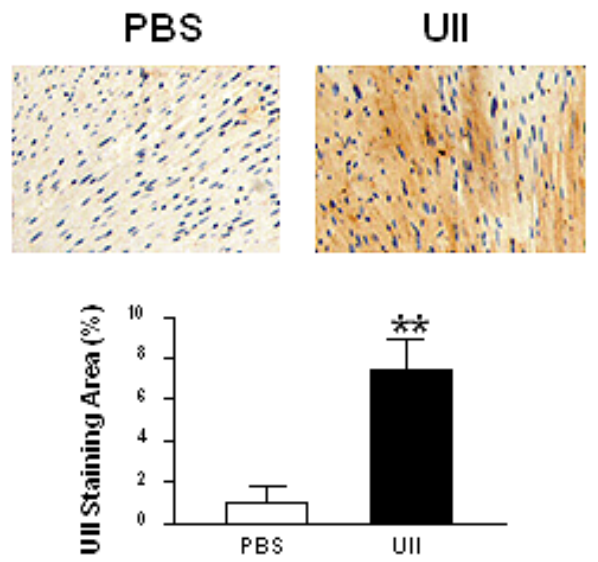

B

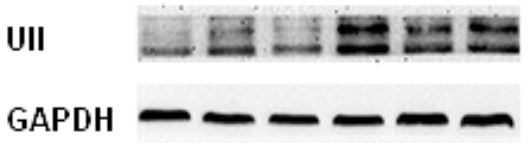

C

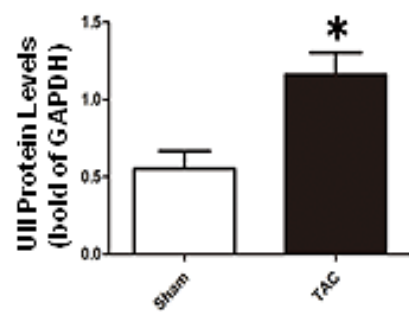

D

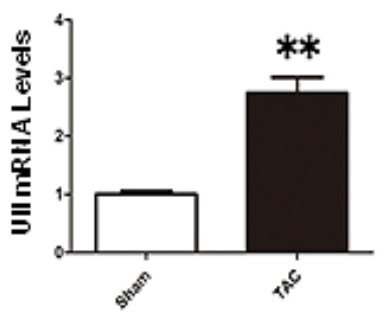

Figure 3. UII expression is elevated in a TAC-induced cardiac hypertrophy mouse model. A: Representative images of UII staining and quantification of UII staining area/total area by immunohistochemical analysis. magnification: $100 \times$. B and C: Quantification of UII protein level (ratio of protein pixel density/GAPDH pixel density) based on immunoglobulin. D: UII mRNA expression in sham-operated or TAC-operated mice. Sham-operated mice: $n=9$, TAC-operated mice: $n=9$. E: Representative images of UII staining and quantification of UII staining area/total area by immunohistochemical analysis in heart tissues of mice with (UII) or without (PBS) UII treatment were shown $(n=$ 3). $* P<0.05, * * P<0.01$

for 48 hours to induce fibrotic responses. The UII receptor antagonist, Urantide (1 $\mu \mathrm{M}$, Peptides International Inc., Louisville, KY, USA), or the same volume of PBS was administered in the medium 30 minutes before UII treatment.

Fibroblast scratch assay: Myocardial fibroblasts were seeded into six-well plates at a density of $1 \times 10^{5}$ cells/ well and cultured for 48 hours at $37^{\circ} \mathrm{C}$. Then, cells were synchronized by fasting in DMEM without FBS for 24 hours. Scratch injury was initiated by making a scratch in the monolayer using a $200 \mu \mathrm{l}$ pipet tip. Cells were treated by UII $(0.1 \mu \mathrm{M})$ for 48 hours with or without Urantide pretreatment. Then, images were taken of the scratch area, and the distance was measured and compared with control cells. Control cells were treated with PBS.

Western blot analysis: Proteins were isolated from frozen heart tissue and cultured cardiac fibroblasts and then extracted using a RIPA buffer (Beyotime, Shanghai, China), containing protease inhibitors and phenylmethylsulfonyl fluoride (PMSF, Beyotime, Shanghai, China). Next, the protein concentration was determined using the bicinchoninic acid assay (BCA) Protein Assay Kit (Thermo). Then, $10 \mu \mathrm{g}$ of each protein sample was separated using SDSPAGE and transferred to polyvinylidene fluoride (PVDF) membranes. After blocking the membranes for 1 hour with PBS with Tween-20 (TBST) containing 5\% bovine serum albumin, membranes were incubated overnight at $4^{\circ} \mathrm{C}$ with antibodies directed against collagen I, TGF- $\beta$, 


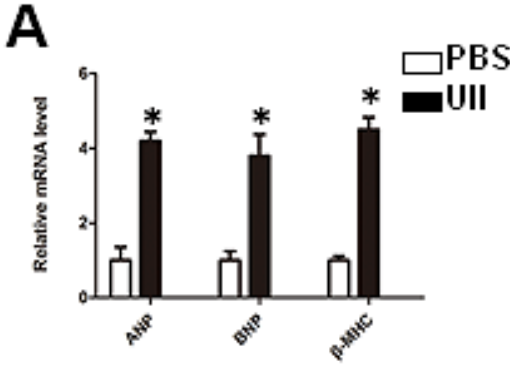

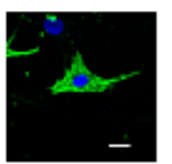

PBS

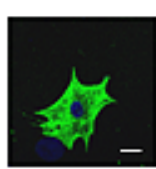

UII
B

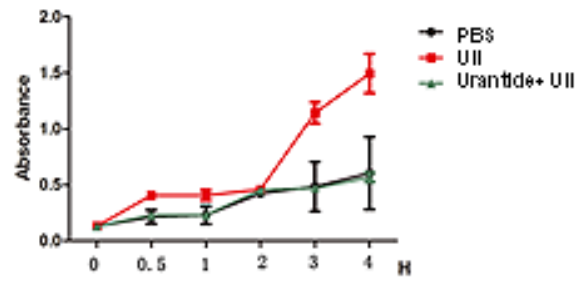

C PBS

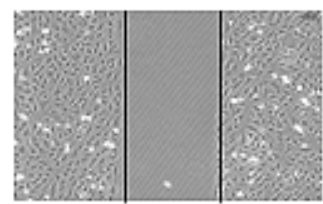

UII

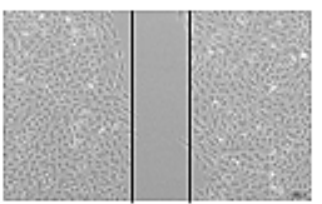

Urantide+UII

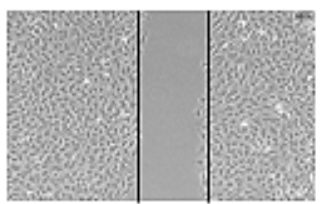

D

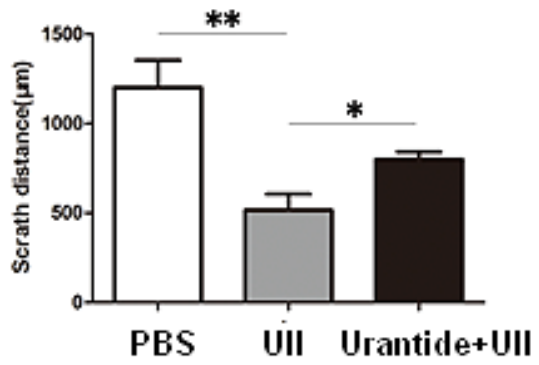

Figure 4. Activation of cardiac fibroblasts induced by UII in vitro. A: mRNA expression of hypertrophic genes $\beta$ - MHC, ANP, and $B N P$ in UII-induced cardiomyopathy. B: Proliferation of cells in the control group, the UII intervention group and the UII + Urantide group was analyzed using the CCK-8 assay. C and D: Representative images of the cell scratch assay of the control group, the UII group, and the UII + Urantide group (magnification: $40 \times$ ). Experiments were performed in triplicate. $* P<0.05, * * P<0.01$

and Phospho-Smad3 (Ser423/425) (1:000 Cell Signaling Technology). Antigen-antibody complexes were visualized using the enhanced chemiluminescence (ECL) method. Horseradish-peroxidase (HRP)-conjugated IgG was used as the secondary antibody (Beyotime, Shanghai, China). Protein levels were quantified using ImageJ software.

Quantitative real-time PCR: Total RNA was extracted from cardiac tissue and cultured cardiac myocytes and fibroblasts using the TRIzol reagent (Invitrogen, Carlsbad, CA USA), and single-stranded complementary DNA was synthesized using a high-capacity complementary DNA reverse transcription kit (Applied Biosystems; ThermoFisher Scientific, Inc., Waltham, MA, USA). For quantitative real-time-PCR (qRT-PCR), the SYBR kit (Tiangen Biotech Co., Ltd., Beijing, China) was used. Gene-specific primers used for qRT-PCR are presented in Table I. GAPDH was used as an internal control. The data are expressed as the between the fold-difference of each gene and GAPDH using the $2^{-\Delta \Delta}$ CT method. The melting point dissociation curve provided with the instrument was used to confirm that only a single product was present. All primers were designed using GenePharma (Shanghai, China) (Table II).

Cell proliferation assay: The CCK- 8 assay was performed using a CCK-8 assay kit (Beyotime, China) according to the manufacturer's instructions. Myocardial fibroblasts were cultured in serum-deprived medium for 24 hours, and $3 \times 10^{3}$ cells were then seeded on gelatincoated 96-well plates and incubated using a CCK8 solution for 24 hours. Absorbance was read using a spectrophotometer (absorbance at $450 \mathrm{~nm}$ ).

Statistical analysis: Data with a normal distribution were expressed as the mean standard error of the mean. For data obtained from a small number of samples, the MannWhitney $U$ test was used between two groups. The Kolmogorov-Smirnov method was used to determine the normal distribution of the other data. Student's $t$ test was employed between two groups, using one-way analysis of variance, whereas Tukey's test was used for comparison of at least three groups. Data were analyzed using SPSS (version 17.0) statistical software. $P<0.05$ was considered statistically significant.

\section{Results}

UII-treated mice showed cardiac hypertrophy and significant cardiac fibrosis: To determine if the UII treatment induces cardiac fibrosis after HCM, a mouse model of UII-induced cardiac hypertrophy was established to represent the above-mentioned cardiac fibrosis response. We demonstrated successful modeling by calculating cardiac weight $(\mathrm{HW})$ to body weight $(\mathrm{BW})$ and HW-tibial length (TL) ratios and using M-mode echocardiography to assess cardiac hypertrophy (Figure 1 and Table I). Compared with those in the PBS-treated control group, mice administrated with UII at $1.5 \mu \mathrm{g} / \mathrm{kg}$ showed a significant increase in total cardiac hypertrophy and higher HW/BW and HW/TL ratios (Figure 1A-D). In the UII group, LV wall thickness (including left ventricular systolic posterior wall (LVPW)) was significantly increased at both the systolic and diastolic levels (Figure 1E), although UII did not induce the elevation of SBP (Table I). For Hematoxylin and eosin (HE) and wheat germ agglutinin (WGA) staining, the hypertrophic phenotype of mice in the UII group was more severe compared to that in PBS-operated mice (Figure 1F). In addition, in the UII group, mRNA levels 
A

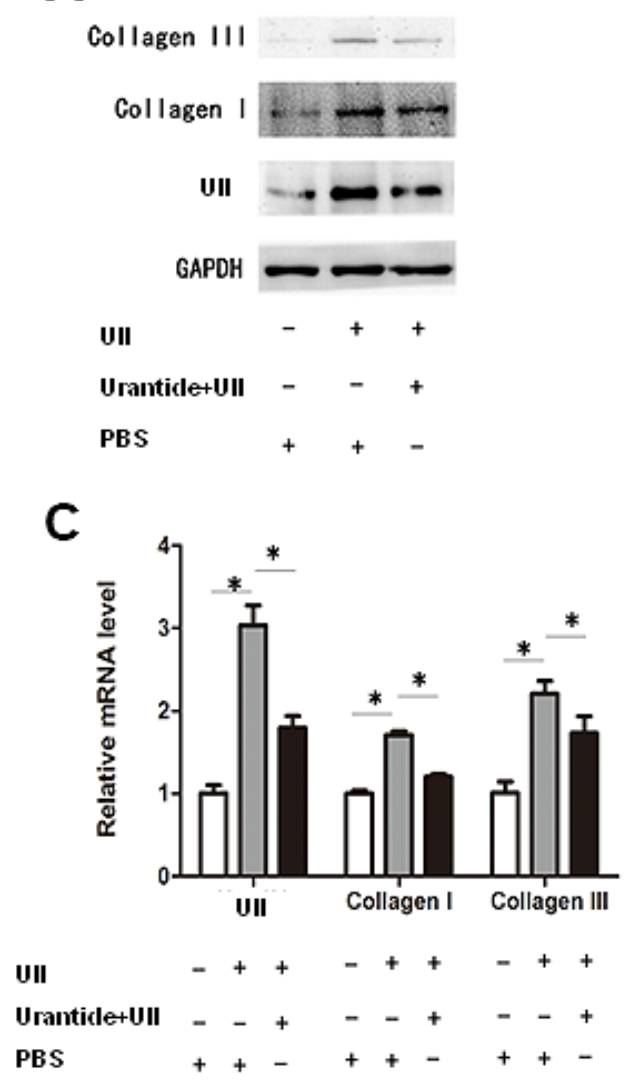

B

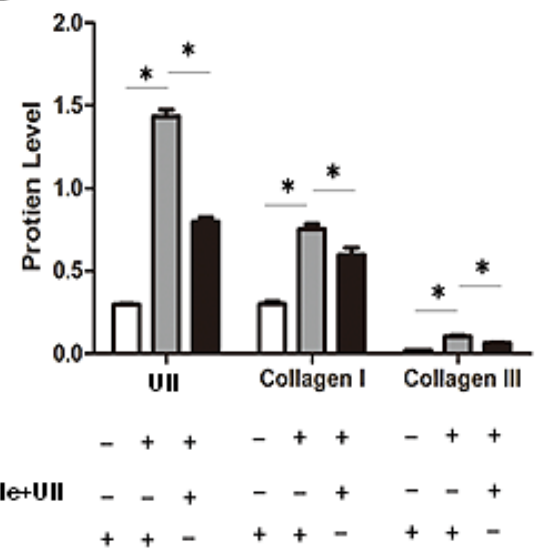

Figure 5. UII induces cardiac fibrosis responses in vitro. A and B: Quantification of UII, collagen I, and collagen III protein levels in PBS-, UII- and UII + Urantide-treated cardiac fibroblasts from the Western blot analysis. C: mRNA expression levels of UII, collagen I, and collagen III in PBS-, UII- and UII + Urantide-treated from RT-PCR. Experiments were performed in triplicate. $* P<0.05$

of ANP, BNP, and $\beta$-MHC were compared with those in PBS-treated mice (Figure 1G), indicating that UII caused cardiac hypertrophy and that the model was successfully established. HE staining showed that myocardial fibrosis in the UII group was more severe compared to that in the control group (Figure 2). To assess cardiac fibrosis, both Masson and SR staining showed more severe fibrosis and interstitial fibrosis in the hearts of UII mice compared to control mice (Figure 2A and B). In addition, Western blot and PCR analyses indicated that collagen I and collagen III were significantly enhanced in mice in the UII-treated group (Figure 2C-E).

UII upregulated in pressure overload-induced mouse heart hypertrophy: To investigate the potential role of UII in hypertrophic cardiac hypertrophy, immunohistochemical staining was performed using an antibody directed against UII. The results indicated increased expression of UII in hypertrophic myocardium in a TAC mouse model (Figure 3A). Western blot analysis and RT-PCR results indicated that in the TAC group, UII was significantly upregulated in the left ventricles of TAC-operated mice compared to mice in the sham-operated control group (Figure 3B-D). These results suggest that UII was upregulated in pressure overload-induced hypertrophy in mice. To compare the levels of UII in the heart between
TAC and UII treatment models, we also examined the cardiac UII level in UII-treated mice using immunohistochemical staining. The results revealed that the cardiac level of UII in mice with UII treatment was higher than that without UII treatment (Figure 3E), which seems to be comparative to that of TAC-treated mice (Figure 3E versus A).

UII regulates cardiac myofibroblast activation in vitro: Cardiomyocytes were isolated from neonatal rats and cultured, and hypertrophy induction was confirmed by an increase in cell-hypertrophy-related gene expression ( $\beta$ MHC, ANP and BNP) after UII treatment (Figure 4A). Cardiac fibroblasts were cultured from neonatal rats and treated with UII. Then, we examined the role of UII in cardiac fibrosis and its underlying mechanisms. UII induced significant migration and proliferation of cardiac fibroblast, an UII receptor antagonist, Urantide inhibited UII-induced cardiac fibroblast migration and proliferation (Figure 4B-D). To further investigate the role of UII in cardiac fibrosis and its underlying mechanisms, UII was added to myocardium fibroblasts to determine whether fibroblasts secrete collagen I and collagen III. Western blot analysis and PCR results indicated that collagen I and collagen III expressions were significantly increased upon UII induction compared to those in the control group 


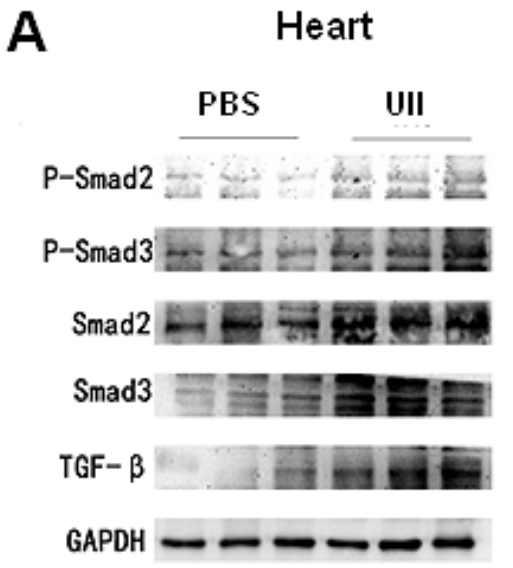

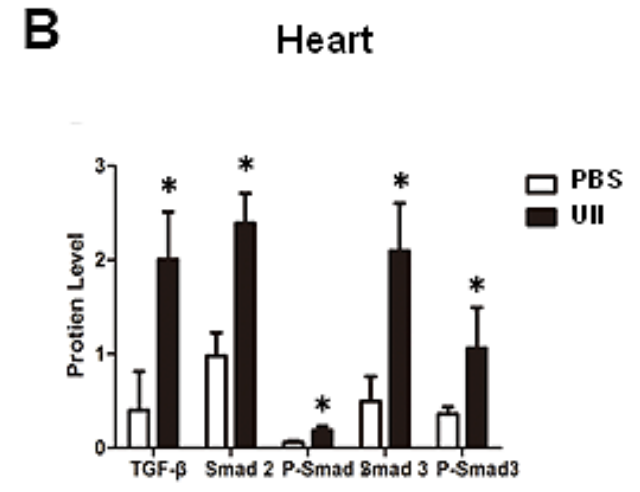

D

C Cardiac Fibroblasts

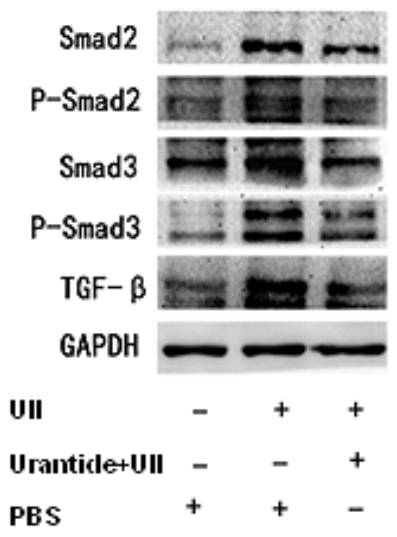

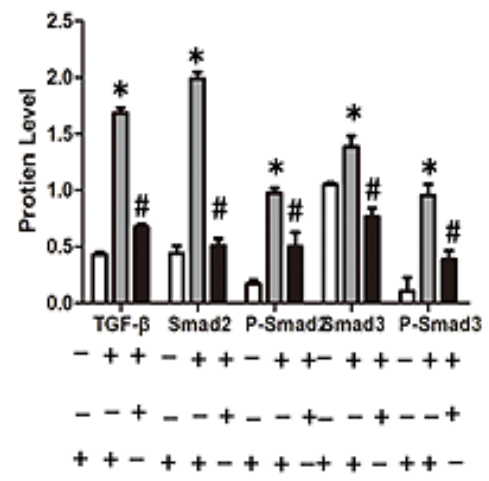

Cardiac Fibroblasts

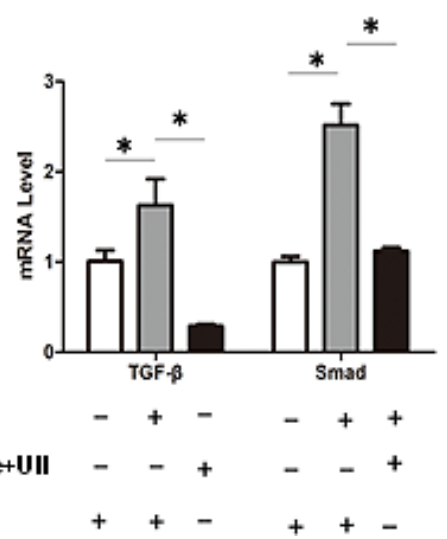

Figure 6. UII activated the TGF- $\beta /$ Smad signaling pathway in cardiac fibrosis. A and B: Activation of TGF- $\beta /$ Smad signaling in vivo was determined in the UII-treated mice and quantified using the Western blot and PCR analyses. C and D: Activation of TGF- $\beta$, Smad2, and Smad3 in PBS-, UII- and UII + Urantide-treated cardiac fibroblasts were evaluated using the Western blot and PCR analyses. In vitro experiments were performed in triplicate. ${ }^{*} P<0.05$. ${ }^{\sharp} P<0.05$, compared between the UII-treated group and the UII + Urantide-treated group.

(Figure 5A-C). Moreover, inhibition of the UII receptor by Urantide inhibited UII-induced collagen I and III expressions (Figure 5A-C).

UII activates TGF- $\beta /$ Smad signaling to regulate fibrotic responses: TGF- $\beta / \mathrm{Smad}$ signaling has been demonstrated to play an important role in the pathogenesis of cardiac remodeling and fibrosis. ${ }^{24-28)}$ The expression of TGF- $\beta$ in the mouse heart is associated with fibrosis and hypertrophy, ${ }^{24-28)}$ and TGF- $\beta /$ Smad signaling has been shown to be a major pathway contributing to both reactive and replacement cardiac fibrosis. ${ }^{24-28}$ Therefore, we evaluated the TGF- $\beta /$ Smad signaling pathway in a UII-treated mouse model. We showed that phosphorylation levels of TGF- $\beta$ and phosphorylation and non-phosphorylation of Smad2 and Smad3 were significantly increased in mice in the UII group (Figure 6A and B) when compared to control mice. As expected, in vitro studies demonstrated that UIIinduced TGF- $\beta$ production was significantly increased, whereas phosphorescent and non-phosphorylated Smad2 and Smad3 levels were highly consistent with TGF- $\beta$ (Figure 6C and D). After adding an inhibitor for the UII receptor to UII-treated cardiac fibroblasts, we demonstrated that TGF- $\beta$ and phosphorescent and nonphosphorylated Smad2 and Smad3 expression were inhibited (Figure 6C and D). Taken together, these results indicated that UII may regulate cardiac fibrosis through the TGF- $\beta /$ Smad signaling pathway.

\section{Discussion}

In our present study, we demonstrated the effect of UII on cardiac fibrosis and underlying mechanisms. The results indicated that (1) UII treatment significantly induced cardiac fibrosis at in vivo and in vitro levels; (2) in TAC-operated mice, the expression of UII was significantly higher than that in the control group; and (3) UIIinduced cardiac fibroblasts might be regulated by the TGF- $\beta /$ Smads signaling pathway.

Although UII plays a critical role in cardiac hypertrophy, the role of UII in the cardiac fibrosis and the underlying regulatory mechanisms are not yet fully understood. Several studies have demonstrated that UII is important 
for the induction and the development of heart failure, and inhibition of UII significantly improved cardiac hypertrophy, cardiac dysfunction, and heart failure. ${ }^{9-13,17,18)}$ Cardiac fibrosis plays an important role in ventricular remodeling and heart failure. ${ }^{24-28}$ It has been reported that UII plays roles in the regulation of cardiac fibrosis; $;^{29,30)}$ however, so far, the direct role of UII in cardiac fibrosis is not clear. Our data confirmed that UII is a direct key fibrous inducer in cardiac fibrosis, which plays a role in cardiac ventricular remodeling and early heart failure. In addition, our study demonstrated that UII promoted cardiac fibrosis by activation of the TGF- $\beta /$ Smads signaling pathway.

TGF- $\beta$ is one of the most important cytokines in the process of fibrosis. ${ }^{24-26)}$ TGF- $\beta$ complexes with TGF- $\beta$ receptors induce Smad2/Smad3 phosphorylation, which affects fibrosis. In UII-induced cardiac fibroblasts, we found a significant increase in TGF- $\beta$ mRNA and protein expression, and an increase of Smad2 and Smad3 protein expression and phosphorylation activity. These two factors are important mediators in the TGF- $\beta$ signaling pathway, and they indicated that in a UII-induced hypertrophic heart, the TGF- $\beta /$ Smads signaling pathway is activated to induce cardiac fibrosis.

To further identify the potential role of UII in fibrosis, the UII receptor antagonist Urantide was used in UIIinduced cardiac fibroblasts. Increases in fibrotic responses and TGF- $\beta$ signaling after UII treatment were greatly attenuated by Urantide. As a downstream protein, the levels of phosphorescent Smad 2, Smad 3, and nonphosphorylated Smad 2, Smad 3 were also reduced. Our data confirmed a relationship between UII and myocardial fibrosis, which may be linked through the TGF- $\beta / \mathrm{Smad}$ signaling pathway. Moreover, in another study, we found that UII could induce cardiomyocyte apoptosis and pyroptosis (data not presented), suggesting that UII might be involved in the development of cardiac remodeling through cardiomyocyte death besides the effect on proliferation of cardiac fibroblasts.

In a previous study, Zhang, et al. demonstrated the changes of UII levels before and after PCI among patients with AMI, and the UII level was a predictor of post-AMI death. ${ }^{18)}$ However, our data confirmed that the UII was elevated in cardiac fibrosis, which provided a possible basis for inhibition of cardiac fibrosis by the UII antagonist. This is of great value in clinical treatment, and improvement of hypertrophic cardiomyopathy may, therefore, provide a potential therapeutic target for cardiac fibrosis.

In conclusion, our findings demonstrated that UIIinduced cardiac fibrosis through TGF- $\beta /$ Smad signaling during the development of cardiac remodeling. By inhibition of the UII receptor, the relevant molecular protein in the TGF- $\beta /$ Smad signaling pathway was inhibited. These findings suggest that UII may be a promising therapeutic target for the prevention and treatment of hypertrophic heart disease-induced cardiac fibrosis.

\section{Disclosure}

Conflicts of interest: The authors confirm that there are no conflicts of interest.

\section{References}

1. Dominguez-Rodriguez A, Abreu-Gonzalez P. Hypertrophic cardiomyopathy. Lancet 2013; 381: 1456-7.

2. Hershberger RE, Cowan J, Morales A, Siegfried JD. Progress with genetic cardiomyopathies: screening, counseling, and testing in dilated, hypertrophic, and arrhythmogenic right ventricular dysplasia/cardiomyopathy. Circ Heart Fail 2009; 2: 253-61.

3. Moreno V, Hernández-Romero D, Vilchez JA, et al. Serum levels of high-sensitivity troponin T: a novel marker for cardiac remodeling in hypertrophic cardiomyopathy. J Card Fail 2010; 16: 950-6.

4. Maron BJ. Hypertrophic cardiomyopathy: a systematic review. JAMA 2002; 287: 1308-20.

5. Pearson D, Shively JE, Clark BR, et al. Urotensin II: a somatostatin-like peptide in the caudal neurosecretory system of fishes. Proc Natl Acad Sci USA 1980; 77: 5021-4.

6. Ames RS, Sarau HM, Chambers JK, et al. Human urotensin-II is a potent vasoconstrictor and agonist for the orphan receptor GPR14. Nature 1999; 401: 282-6.

7. Coulouarn Y, Jégou S, Tostivint H, Vaudry H, Lihrmann I. Cloning, sequence analysis and tissue distribution of the mouse and rat urotensin II precursors. FEBS Lett 1999; 457: 28-32.

8. Bottrill FE, Douglas SA, Hiley CR, White R. Human urotensinII is an endothelium-dependent vasodilator in rat small arteries. Br J Pharmacol 2000; 130: 1865-70.

9. Pereira-Castro J, Brás-Silva C, Fontes-Sousa AP. Novel insights into the role of urotensin II in cardiovascular disease. Drug Discov Today 2019; 24: 2170-80.

10. Stirrat A, Gallagher M, Douglas SA, et al. Potent vasodilator responses to human urotensin-II in human pulmonary and abdominal resistance arteries. Am J Physiol Heart Circ Physiol 2001; 280: H925-8.

11. Totsune K, Takahashi K, Arihara Z, Sone M, Ito S, Murakami O. Increased plasma urotensin II levels in patients with diabetes mellitus. Clin Sci 2003; 104: 1-5.

12. Russell FD. Emerging roles of urotensin-II in cardiovascular disease. Pharmacol Ther 2004; 103: 223-43

13. Nakayama T, Hirose T, Totsune $\mathrm{K}$, et al. Increased gene expression of urotensin II-related peptide in the hearts of rats with congestive heart failure. Peptides 2008; 29: 801-8.

14. Hirose T, Takahashi K, Mori N, et al. Increased expression of urotensin II, urotensin II-related peptide and urotensin II receptor mRNAs in the cardiovascular organs of hypertensive rats: comparison with endothelin-1. Peptides 2009; 30: 1124-9.

15. Mori N, Hirose T, Nakayama T, et al. Increased expression of urotensin II-related peptide and its receptor in kidney with hypertension or renal failure. Peptides 2009; 30: 400-8.

16. Katano Y, Ishihata A, Aita T, Ogaki T, Horie T. Vasodilator effect of urotensin II, one of the most potent vasoconstricting factors, on rat coronary arteries. Eur J Pharmacol 2000; 402: R5-7.

17. Jumaah S, Çelekli A, Sucu M. The role of human urotensin-II in patients with hypertrophic cardiomyopathy. J Immunoassay Immunochem 2018; 39: 150-62.

18. Zhang X, Ye Y, Yang C, et al. Clinical significance of increased urotensin II levels in acute myocardial infarction. Discov Med 2018; 26: 7-20.

19. Gong H, Ma H, Liu M, et al. Urotensin II inhibits the proliferation but not the differentiation of cardiac side population cells. Peptides 2011; 32: 1035-41.

20. Chen Z, Xu J, Ye Y, et al. Urotensin II inhibited the proliferation of cardiac side population cells in mice during pressure overload by JNK-LRP6 signalling. J Cell Mol Med 2014; 18: $852-62$.

21. Zhang YG, Li YG, Liu BG, et al. Urotensin II accelerates cardiac fibrosis and hypertrophy of rats induced by isoproterenol. Acta Pharmacol Sin 2007; 28: 36-43.

22. Zou Y, Liang Y, Gong H, et al. Ryanodine receptor type 2 is required for the development of pressure overload-induced cardiac hypertrophy. Hypertension 2011; 58: 1099-110. 
23. Zou Y, Komuro I, Yamazaki T, et al. Cell type-specific angiotensin II-evoked signal transduction pathways: critical roles of Gbetagamma subunit, Src family, and Ras in cardiac fibroblasts. Circ Res 1998; 82: 337-45.

24. Morine KJ, Qiao X, York S, et al. Bone morphogenetic protein 9 reduces cardiac fibrosis and improves cardiac function in heart failure. Circulation 2018; 138: 513-26.

25. Dobaczewski M, Chen W, Frangogiannis NG. Transforming growth factor (TGF)-beta signaling in cardiac remodeling. J Mol Cell Cardiol 2011; 51: 600-6.

26. Yue Y, Meng K, Pu Y, Zhang X. Transforming growth factor beta (TGF-beta) mediates cardiac fibrosis and induces diabetic cardiomyopathy. Diab Res Clin Pract 2017; 133: 124-30.

27. Sniegon I, Priess M, Heger J, Schulz R, Euler G. Endothelial mesenchymal transition in hypoxic microvascular endothelial cells and paracrine induction of cardiomyocyte apoptosis are mediated via TGFbeta(1)/SMAD signaling. Int J Mol Sci 2017; 18: 2290.

28. Zou Y, Cai Y, Lu D, Zhou Y, Yao Q, Zhang S. MicroRNA-146a$5 \mathrm{p}$ attenuates liver fibrosis by suppressing profibrogenic effects of TGF $\beta 1$ and lipopolysaccharide. Cell Signal 2017; 39: 1-8.

29. Dai HY, He T, Li XL, Xu WL, Ge ZM. Urotensin-2 promotes collagen synthesis via ERK1/2-dependent and ERK1/2independent TGF-b1 in neonatal cardiac fibroblasts. Cell Biol Int 2011; 35: 93-8.

30. Liu W, Han Q, Liu Q, Liang G, Wang J, Liu C. An investigation into the expression and mechanism of action of urotensin II in chronic pressure-overloaded rat hearts. Mol Med Rep 2015; 12: 6626-34. 